\section{The functional anatomy of humor: segregating cognitive and affective components}

\author{
Vinod Goel ${ }^{1,2}$ and Raymond J. Dolan ${ }^{1,3}$ \\ 1 Well come Department of Cognitive Neurology, Institute of Neurology, \\ Queens Square, London WC1N 3BG, UK \\ 2 Department Of Psychology, York University, Toronto, Ontario M 3] 1P3 \\ Canada \\ 3 Royal Free Hospital School of M edicine, Roland Hill Street, London NW3, UK \\ Correspondence should be addressed to V.G. (vgoel@yorku.ca) or \\ R.J.D. (r.dolan@fil.ion.udl.ac.uk)
}

Humor, a unique human characteristic, is critical in thought, communication and social interaction. Successful jokes involve a cognitive juxtaposition of mental sets, followed by an affective feeling of amusement; we isolated these two components of humor by using event-related $\mathrm{fM} \mathrm{RI}$ on subjects who listened to auditorily presented semantic and phonological jokes (puns) and indicated whether or not they found the items amusing. Our findings suggest that whereas there are modality-specific pathways for processing the juxtaposition of mental sets necessary for the appreciation of jokes, a common component of humor is expressed in activity in medial ventral prefrontal cortex, a region involved in reward processing.

We scanned 14 right-handed normal subjects using event-related fM RI while they listened to jokes. Thirty semantic jokes of the form, "What do engineers use for birth control?... Their personalities," and thirty phonological jokes or puns of the form, "Why did the golfer wear two sets of pants?... He got a holein one," werepre sented to subjects in arandom order. In corresponding baseline conditions, identical setup lines were used, but the punch lines were modified to be merely descriptive. (For example, "What do engineers use for birth control?... The pill." "Why did the golfer wear two sets of pants? ... It was a very cold day.") Items were pre recorded, balanced across conditions for the number of phonemes and temporal duration, and auditorily presented to subjects in random order (Fig. 1). While in the scanner, subjects were requested to make judgments (recorded by a key press) as to whether or not they found each item amusing. After the scan, subjects reviewed the jokes and rated them for 'funniness' on a scale of 1 to 5. The study was approved by the Joint National Hospital for Neurology and Neurosurgery/Institute of Neurology Ethics Committee. Data were analyzed using statistical parametric mapping (SPM 99) ${ }^{1}$ using a random effects model.

Subjects took longer $\left(t_{29}=7.18, p<0.0001\right)$ to respond to jokes (mean \pm s.d., $2824 \pm 259 \mathrm{~ms}$ ) after thepresentation of the punch line than to non-jokes ( $2550 \pm 220 \mathrm{~ms}$ ). Therewas no differencein response timesto jokes as a function of funniness ratings. While being scanned, subjects rated $62 \pm 16 \%$ of jokes as 'amusing,' with no significant difference between the ratings of semantic and phonological jokes.

To isolate mechanisms underlying juxtaposition of mental sets, we examined the main effect of jokes ( (semantic jokes + phonological jokes) - (semantic baseline + phonological baseline)). This comparison revealed activation in left posterior middle temporal gyrus (BA 21/37), left posterior inferior temporal gyrus (BA 37), right posterior middle temporal gyrus (BA 21) and left inferior frontal gyrus (BA 44, 45). H owever, an analysis of simple main effects and direct comparisons of semantic and phonological joke conditions revealed that juxtaposition of semantic and phonological mental sets require different networks. The comparison of semantic jokes with the corresponding non-joke baselinerevealed activation in left posterior middletemporal gyrus (BA 21/37), left posterior inferior temporal gyrus (BA 37) and right posterior middletemporal gyrus (BA 21; Fig. 2a). The comparison of puns with their corresponding non-jokebaselines revealed activation in left posterior inferior temporal gyrus (BA 37) and left inferior frontal gyrus (BA 44, 45; Fig. 2b). The direct comparison of semantic and phonological jokes (masked by the main effect of jokes; (semantic + phonological jokes) - (baselines) ) revealed an anatomical dissociation. Semantic jokes compared to phonological jokes (semantic jokes - phonological jokes) recruited the right posterior middle temporal gyrus (BA 21/37) and left posterior inferior temporal gyrus (BA 20/37) (Fig. 2c). The reverse comparison (phonological jokes - semantic jokes) revealed activation of left insula/precentral gyrus (Fig. 2d).

The activation of posterior temporal lobein semantic jokes-a region involved in semantic processing of language - highlights the importance of differential semantic processing in the joke punch line over and abovethenon-jokeitem. The involvement of theright temporal lobeis consistent with several studies that have implicated it in processing and maintaining alternative, less probable word meanings, and in integrative processes requiring global coherence ${ }^{2-5}$. These processes are necessary for semantic juxtapositions of meaning required to 'get' the punch line. Similarly, activation of left inferior prefrontal cortex and insula - regions involved in processing of

Fig. 1. Subjects were presented with semantic and phonological jokes while undergo ing fMRI scans. The stimuli were prerecorded as voice files, edited to precise temporal lengths and presented in a random order, in a time-locked single-event design. The setup line began at time 0 and had a duration ranging from 1340 to $3230 \mathrm{~ms}$ (mean duration, $2420 \mathrm{~ms}$ ). A mean pause of $1580 \mathrm{~ms}$ gave subjects time to consider the setup line. At $4000 \mathrm{~ms}$, the punch line (or descriptive answer) was delivered. It lasted 480 to $3250 \mathrm{~ms}$ (mean, $1540 \mathrm{~ms}$ ). Thereafter, subjects had a variable amo unt of time to respond. In all trials, subjects were asked to provide a subjective judgment (by pressing a button on a keypad) of whether they found the item amusing or not. Subjects were instructed to refrain from laughter to minimize head movement. A 2T Siemens VISION system (Siemens, Erlangen, Germany) was used to acquire $48,1.8-\mathrm{mm}$ thick (with $1.2 \mathrm{~mm}$ gap), T2*-weighted echoplanar images (64 $\times$ $64,3 \times 3 \mathrm{~mm}$ pixels; TE, $40 \mathrm{~ms}$ ) of the whole brain sensitive to blood oxygenation level dependent (BO LD) contrast. Data was recorded in one 18.1 min session of 120 events using a TR of $4.1 \mathrm{~s}$ and random jitter of 1 TR. The BO LD signal was sampled as a hrf at the midpoint between the presentation of the punch line and the subjects' motor response.

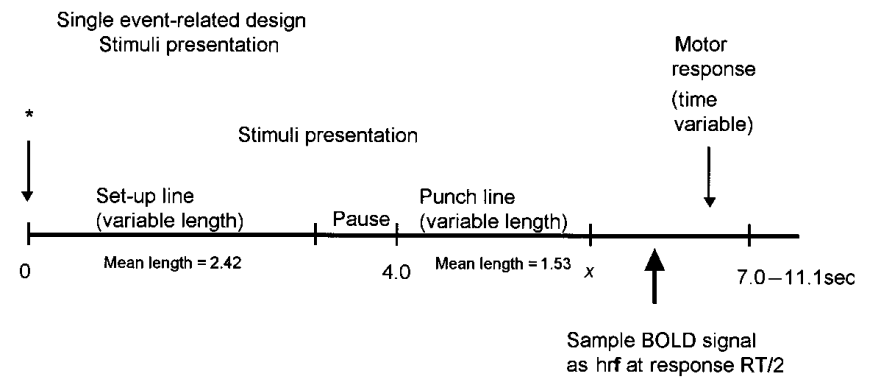

as hrf at response RT/2 

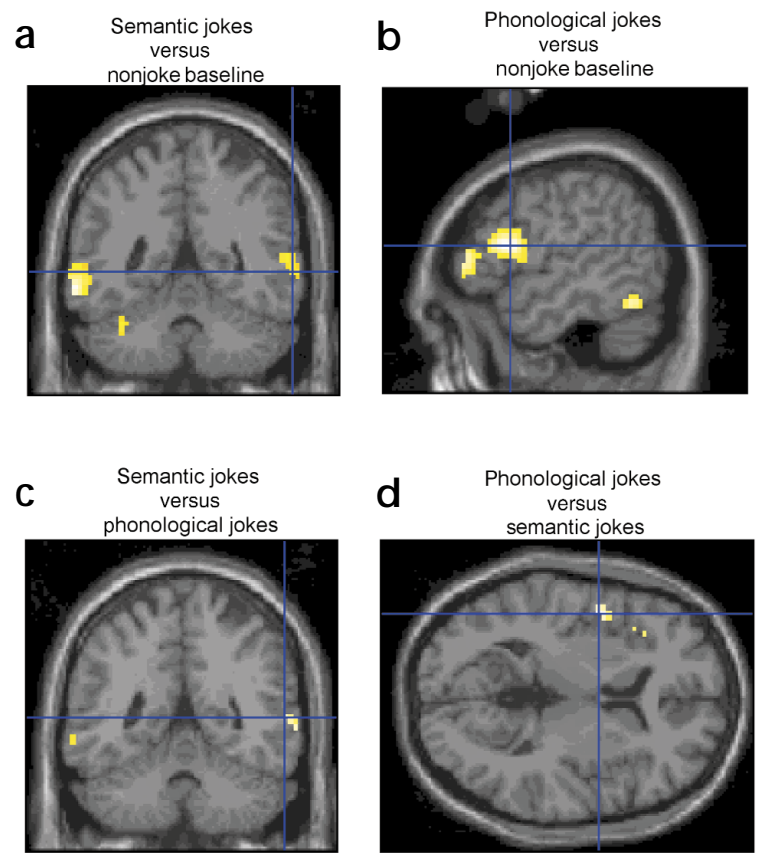

\section{e Affective component} of successful jokes

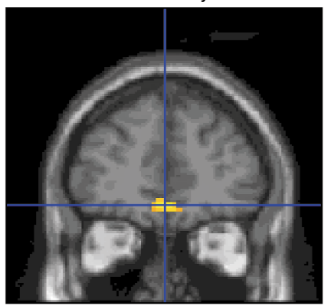

f

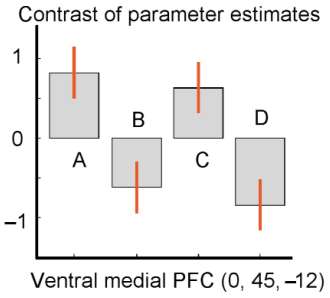

Fig. 2. An anatomical dissociation occurred in the cognitive processing of semantic and phonological jokes, whereas a common region underlay affective processing. (a) Processing of semantic jokes activated left posterior middle temporal gyrus (BA 21/37; $-60,-54,0 ; Z=4.65$ ), left posterior inferior temporal gyrus $(-63,-51,-9 ; Z=4.81)$, right posterior middle temporal gyrus (BA 21; 63, $-48,0 ; Z=3.21$ ) and cerebellum $(36,-63,-30 ; Z=$ 4.81 and $-36,-54,-33 ; Z=4.68)$. (b) Processing of phonological jokes activated left posterior inferior temporal gyrus (BA 37; -60,-54,-12; Z = 5.34 and $-48,-57,-21 ; Z=5.08$ ), and left inferior frontal gyrus (BA 44, 45; -51 , 15,$15 ; Z=5.32$ and $-54,36,6 ; Z=4.93$ ). (c) A direct comparison of semantic jokes with phonological jokes revealed activation in right posterior middle temporal gyrus (BA 21/37; 66, $-45,0 ; Z=5.01$ and $60,-48,6$; $Z=4.84$ ) and left posterior inferior temporal gyrus (BA 20/37; $-63,-39$, -15; $Z=5.19)$. (d) The reverse comparison revealed activation of left inferior precentral gyrus/insula (BA 44/4; $-48,0,6 ; Z=4.71$ ). (e) Activation in $\operatorname{MVPFC}(0,45,-12 ; Z=4.67)$ and bilateral cerebellum $(27,-72,-27 ; 15$, $-93,-36 ;-48,-72,-33$; data not shown) was common to both types of humor. 0 nly activity in the MVPFC $(3,48,-12 ; Z=4.73)$ correlated with subjects' post-scan funniness ratings of jokes. (f) Condition-specific parameter estimates show that the MVPFC responded very similarly to both types of funny and not funny jokes. $A$, semantic funny; $B$, semantic not funny; $C$, pun funny; $D$, pun not funny.

frontal cortex in humor appreciation ${ }^{11}$. However, the neuroanatomical evidence indicated that most impaired patients also had damage to medial prefrontal cortex, a finding consistent with our results. Other patient studies have implicated the right hemisphere more generally, and have used semantic jokes ${ }^{12}$. O ur results suggest that what these studies may be capturing is the right temporal lobe's involvement in theintegrativesemantic processes requiring global coherence, necessary for juxtaposition of mental sets, rather than the affective component of amusement $5,14,15$.

In summary, we present evidencefor differential systems underlying the cognitiveand affective processing of humor. Furthermore, the cognitive processing associated with thejuxtaposition of phonological and semantic mental sets is type specific, whereas a common, modality-independent network underwrites the affective component of humor. Semantic juxtaposition uses a bilateral temporal lobe network, whereas phonological juxtaposition uses a left hemisphere network centered around speech production regions. The affective appreciation of humor involves access to a central reward system in M VPFC.

\section{ACKNOWLEDGEMENTS}

V.G. is supported by a M CD onnell-Pew Program in Cognitive Neuroscience Award, and NSERC and SSH RC grants. R.J.D. is supported by the Wellcome Trust. The authors acknowledge advice from B. Strange and R. H enson. cognitive processing associated with juxtaposition of the punch line - because this needed to occur in both cases - and highlighted differences due to the pleasurable affect that was associated with funny jokes, and absent in non-funny jokes. Subsequently, weundertook a conjunction analysis, masked by the main effect of humor (at $p=0.05$ ), of activations associated with semantic and phonological funny jokes (conjunction (subjectively funny semantic jokes - subjectively non-funny semantic jokes), (subjectively funny phonological jokes - subjectively non-funny phonological jokes)) to highlight regions activated in common across these two categories. This analysis revealed significant activation in medial ventral prefrontal cortex (M VPFC; BA 10/11) alone and bilateral cerebellum (Fig. 2e). Furthermore, the activation in M VPFC covaried with the subjects' post-scan ratings of joke funniness ( $p<0.05$, corrected). The activation in MVPFC during jokeappreciation, and its covariation with subjectivefunniness ratings, was striking in that it involved a region that represents and controls reward-related behaviors ${ }^{8-10}$.

Few lesion studies of humor appreciation exist ${ }^{11-13}$. A study involving frontal lobepatientsimplicated the right dorsolateral pre-

\section{Received 25 August; Accepted 28 December 2000}

1. Friston, K. et al. Hum. Brain M app. 2, 189-210 (1995).

2. Coney, J. \& Evans, K. D. Neuropsychologia 38, 272-282 (2000).

3. Faust, M . \& Chiarello, C. Neuropsychologia 36, 827-835 (1998).

4. Federmeier, K. D. \& Kutas, M. Brain Res. Cogn. Brain Res. 8, 373-392 (1999).

5. St. George, M ., Kutas, M ., M artinez, A. \& Sereno, M. I. Brain 122, 1317-1325 (1999)

6. Dronkers, N. F. Nature 384, 159-161 (1996)

7. Wise, R. J., Greene, J., Buchel, C. \& Scott, S. K. Lancet 353, 1057-1061 (1999).

8. Rolls, E. T. Cereb. Cortex 10, 284-294 (2000).

9. Anderson, S. W., Bechara, A., Damasio, H., Tranel, D. \& Damasio, A. R. Nat Neurosci 2, 1032-1037 (1999).

10. Schultz, W., Tremblay, L. \& Hollerman, J. R. Cereb. Cortex 10, 272-284 (2000).

11. Shammi, P. \& Stuss, D. T. Brain 122, 657-666 (1999)

12. Wapner, W., Hamby, S. \& Gardner, H. Brain Lang. 14, 15-33 (1981)

13. Gardner, H., Ling, P. K., Flamm, L. \& Silverman, J. Brain 98, 399-412 (1975).

14. Brownell, H. H., Potter, H. H., M ichelow, D. \& Gardner, H. Brain Lang. 22, 253-265 (1984)

15. Brownell, H. H., Simpson, T. L., Bihrle, A.M ., Potter, H. H. \& Gardner, H. Neuropsychologia 28, 375-383 (1990) 